\title{
POUR UNE HISTOIRE DE LA PSYCHOLOGIE
}

L'histoire de la psychologie, telle qu'elle apparaît dans quelques ouvrages (E.G. Boring, $1950^{1}$; M. Reuchlin, $1957^{2}$; P. Fraisse et J. Piaget, $1963^{3}$ ) ou dans les chapitres introductifs de quelques manuels (M. Reuchlin, $\left.1977^{4}\right)$, reflète une adhésion, rarement discutée, à une conception internaliste. La psychologie serait, selon cette conception, animée par une dynamique propre, un processus évolutif totalement endogène et serait indépendante des facteurs externes tels les domaines religieux, socio-politiques et économiques. Tout au plus, les tenants de cette histoire acceptent-ils de voir la psychologie influencée par des disciplines qui se situent à ses frontières comme la biologie, la physiologie et, dans une moindre mesure, la physique. Ces domaines frontières concernent généralement les processus ou les objets psychologiques habituellement qualifiés d'inférieurs comme les réflexes, les sensations et perceptions par opposition au langage et à la pensée qualifiés de processus supérieurs. Ces mêmes domaines frontières ont à leur tour engendré des sous-domaines relativement autonomes telles la psychophysique ou la psychophysiologie.

Corrélativement à cette conception internaliste, le développement scientifique est présenté comme un cheminement vers l'état de psychologie positive tel qu'il fut défini par A. Comte, en 1837, dans la 45' leçon du Cours de philosophie positive ${ }^{5}$. La marche vers la positivité était indiquée par A. Comte lui-même : étude de l'anatomo-physiologie du

1. Edwin G. Boring, A History of Experimental Psychology, New York, Appleton, 1929 et 1950.

2. Maurice Reuchlin, Histoire de la psychologie, Paris, P.U.F., 1957.

3. Paul Fraisse, Jean PIAGET, Traité de psychologie expérimentale. T. 1 : Histoire et méthode, Paris, P.U.F., 1963.

4. M. Reuchlin, Psychologie, Paris, P.U.F., 1977.

5. Auguste COMTE, Philosophie première ou: Cours de philosophie positive, Paris, Hermann, 1975.

Revue de synthèse: IVe S. Nos 3-4, juil.-déc. 1988. 
système nerveux (la phrénologie de Gall lui semblera, à cet égard, un apport décisif), études comparatives, analyse des cas pathologiques, étude des comportements animaux et étude du développement individuel. A voir l'évolution de la psychologie sur les cent dernières années, on serait tenté de soutenir qu'elle a réalisé le projet positiviste. Psychologie différentielle, psychopathologie, éthologie animale et psychologie de l'enfant sont autant de domaines qui concourent à cette réalisation. Cependant, ces domaines sont loin d'apparaître homogènes en regard des méthodes employées et des modèles épistémologiques auxquels ils se réfèrent. Un examen attentif de ces dimensions méthodologiques et épistémologiques montre plutôt qu'on avance en ordre dispersé. C'est que les développements les plus décisifs de la psychologie sont souvent provoqués par des concepts ou des théories importés d'autres disciplines. Pour ne citer que quelques exemples parmi les mieux connus, rappelons simplement l'influence déterminante du darwinisme sur le behaviorisme de Watson et de Skinner, celle, non moins décisive, de la pensée religieuse sur les choix épistémologiques fondamentaux de J. Piaget (même si celui-ci l'a gommée dans les récits autobiographiques qu'il nous a donnés) ${ }^{6}$ et enfin celle de l'avènement de l'informatique qui constitue la métaphore sur laquelle repose l'essentiel du discours cognitiviste.

La propagation du mythe d'une fondation de la psychologie - qui lui tient lieu d'histoire - a eu pour fonction principale d'assurer à celle-ci sa légitimité. Il s'agit, tout d'abord, d'accorder à la psychologie le statut de science en donnant à ce terme le sens limité que lui confère le positivisme de Comte. Il s'agit, ensuite, de donner à la psychologie son autonomie. Quoi de mieux, pour assurer celle-ci, qu'un mythe de rupture : la psychologie acquerrait, péniblement, son autonomie au terme d'un long combat avec la philosophie. Cette rupture mythique permet à quelques personnages de faire figure de pères fondateurs, créant ex nihilo la nouvelle discipline. Dans cette recherche en paternité, Wundt et Freud jouiront de ce statut prestigieux mais aussi Fechner, dans une moindre mesure, à condition de ne retenir de son cuvre que les Elemente der Psychophysik (1860) ${ }^{7}$. Lorsque Fechner est appelé à figurer au

6. Les principaux ouvrages religieux de J. PIAGeT sont : La Mission de l'idée, Lausanne, La Concorde, 1916; La Psychologie et les valeurs religieuses, Genève, Labor, 1923 et (en coll. avec Jean DE LA HARPE) Deux types d'attitudes religieuses. Immanence et transcendance, Genève, Labor, 1928. J. Piaget évoque ses sentiments religieux dans Sagesse et illusion de la philosophie, Paris, P.U.F., 1965, p. 12.

7. Gustav Theodor FeCHNER, Elemente der Psychophysik, Leipzig, Breitkopf et Hărtel, 1860,2 vols. Le premier volume a fait l'objet d'une traduction anglaise par Helmut $E$. ADLER, Elements of Psychophysics, New York, Holt, Rinehart and Wilson, Inc., 1966. 
nombre des pères fondateurs, la référence à Comte ne convient plus : tout porte à croire, en effet, que Fechner ne se réfère pas au courant comtien. C'est plutôt, dans son cas, le mythe d'une influence de l'œuvre de Christian Wolff que l'on développe. C. Wolff était un partisan de l'Aufklärung et fut même expulsé, en 1723, de l'université de Halle pour excès de rationalisme; son ambition était de légiférer sur la Nature de l'homme, comme Newton l'avait fait sur le monde cosmique, mais en maintenant la distinction fondamentale entre l'âme et le corps, entre univers spirituel et univers matériel. L'objectif de la Psychologia empirica $(1732)^{8}$ est de déterminer les règles et les lois de la pensée comme la physique détermine celles du mouvement des corps. Cette similitude se prolonge même jusqu'à l'exigence de la mesure et de la modélisation mathématique que traduit le concept de "Psycheometria " : "Theoremata haec ad Psycheometriam pertinent, quae mentis humanae cognitionem mathematicam tradit et adhuc in desideratis est $"$.

Or, au contraire, Fechner est un savant romantique, élève de L. Oken, le naturaliste, admirateur et biographe de Jakob Böhme. Le premier exposé de ses conceptions en matière de psychophysique se trouve dans un traité de métaphysique qu'il publie en 1851 : Zend Avesta, oder über die Dinge des Himmels und des Jenseits ${ }^{10}$. Il y exprime nettement un monisme spiritualiste :

« $\mathrm{j}$ 'ai antérieurement soutenu contre l'opinion commune que les plantes ont une âme; je soutiens aujourd'hui qu'il en est de même des astres, avec cette différence que la vie spirituelle des astres est supérieure, celle des plantes inférieure à la nôtre " $" 1$.

De même Wundt, qui est souvent rattaché à cette même lignée, est plutôt le continuateur de Johannes Müller et de Hermann Lotze et s'efforcera de se maintenir sur la voie étroite qui sépare la mythobiologie des Romantiques et le positivisme scientiste, le monisme panthéiste et le monisme matérialiste.

Inscrire Fechner ou Wundt dans une lignée dont Wolff serait l'origine, leur attribuer les mêmes intentions sous prétexte qu'ils eurent les mêmes exigences de mesure que Wolff, voilà comment on construit le mythe de la rupture d'avec la philosophie.

8. Christian WolfF, Psychologia empirica, Francfort, Renger, 1732. Nous utilisons la réédition latine qu'en a donnée Jean ÉCOLE, Hildesheim, 1968.

9. Ibid., $\$ 522$ : «Ces théorèmes appartiennent à la Psychométrie qui procure une connaissance mathématique de l'esprit humain et est encore très attendue. "

10. G. T. FeChNER, Zend Avesta, oder uber die Dinge des Himmels und des Jenseits, vom Standpunkt der Naturbetrachtung, 2 vols, Hambourg, Voss, 1851.

11. Ibid., t. I, p. 1. 
Si rupture il y a à l'époque, elle doit plutôt être attribuée à l'impact de la conception évolutionniste sur la psychologie. D'une part, en effet, la proclamation darwinienne de l'animalité de l'homme (postulat d'ascendance commune) et la définition de la seule contingence comme moteur de l'évolution devait conduire la psychologie sur la voie de la radicalisation de la thèse empiriste et l'orienter vers le behaviorisme. Certes, on s'est plu à voir en Darwin le fondateur de la psychologie comparée ou même encore celui de la psychologie de l'enfant mais c'est surtout la psychologie du comportement, le behaviorisme, qui s'est nourrie du darwinisme en transposant le mécanisme darwinien de l'évolution des espèces au niveau de l'évolution des comportements chez un individu : l'environnement sélectionne les comportements comme il sélectionne les phénotypes. Cette perspective a connu les succès que l'on sait.

Mais, d'autre part, nombreux sont les psychologues qui ont rejeté le darwinisme. Préférant maintenir des schémas explicatifs fondés sur le mouvement et la finalité, ils ont assuré la continuité des conceptions néo-aristotéliciennes de Trendelenburg ${ }^{12}$ et de Brentano ${ }^{13}$ dont l'essentiel des concepts, en matière de psychologie, a été repris par l'école de la "Gestaltpsychologie " ${ }^{14}$. Le primat de l'activité, la finalité du développement, la dynamique des relations entre la totalité et les parties, concepts qui participent de ce courant, sont les caractéristiques essentielles de la pensée piagétienne, par exemple, énoncées en même temps que le rejet de l'empirisme et du darwinisme. Dans cette même orientation, on trouve aussi le concept de "pensée productive", développé par M. Wertheimer $(1945)^{15}$, qui est largement emprunté à l'interprétation qu'a donnée Brentano du « nous poièticos " ${ }^{16}$ d'Aristote et se situe au point d'articulation de la Psychologie de la Forme et du cognitivisme contemporain. Ces psychologues, qui se sont montrés réservés à l'égard du darwinisme, ne se sont pas privés de référence à la biologie mais ils ont choisi leurs modèles en dehors des conceptions évolutionnistes. Et c'est l'embryologie qui apparaît comme leur source d'inspiration la plus fréquente et cela d'autant plus que, sur le plan

12. Friedrich Trendelenburg, Logische Untersuchungen, Leipzig, Hirzel, $2^{\bullet}$ éd. 1870 , 2 vols.

13. Franz Brentano, Psychologie du point de vue empirique, Paris, Montaigne, 1944.

14. Kurt LEWIN, Psychologie dynamique. Les relations humaines, Paris, P.U.F., 1959, cf., en part., le chapitre introductif, « Le conflit entre les modes de pensée galiléen et aristotélicien dans la psychologie contemporaine $»$.

15. Max WertheIMER, Productive Thinking, New York, Harper, 1945.

16. F. Brentano, Die Psychologie des Aristoteles, insbesondere seine Lehre vom nous poietikos, Mayence, Kircheim, 1867. 
épistémologique, elle s'apparente d'assez près, au moment de sa constitution, à l'organicisme. L'épigenèse a toujours fait bon ménage avec le vitalisme et marquera de son empreinte la psychologie de l'enfant. Les premiers psychologues de l'enfance sont d'ailleurs des physiologistes, comme A. Kussmaul ${ }^{17}$, ou des embryologistes, comme W. Preyer ${ }^{18}$. Cette psychologie sera marquée de l'empreinte de la loi de récapitulation (l'ontogenèse est une brève récapitulation de la phylogenèse) popularisée par Haeckel ${ }^{19}$, reprise et généralisée par J. Piaget. Un examen des sources biologiques de ce dernier, tel qu'on peut le faire à la lecture de son Introduction à l'épistémologie génétique ${ }^{20}$, montre que sont dominantes les références à l'embryologie expérimentale et, dans une moindre mesure, à l'évolutionnisme non darwinien. On peut, enfin, faire remarquer que la philosophie biologique qui se construit autour des travaux des embryologistes, celle de $\mathrm{H}$. Driesch ${ }^{21}$ par exemple, à la fin du XIX siècle, mais aussi celle de $\mathrm{P}$. Weiss ${ }^{22}$ au milieu du $\mathrm{XX}^{e}$ siècle, fait grand usage de concepts comme totalité, régulation, interaction, finalité, émergence, etc., c'est-à-dire des concepts cardinaux du cognitivisme contemporain.

D'une certaine façon, l'avènement du darwinisme produit bien une rupture à l'intérieur du discours de la psychologie, mais la référence de la psychologie à la biologie, que cet avènement impose, divise plutôt qu'elle ne rassemble. L'inspiration biologique, tant sur le plan conceptuel que méthodologique, n'est donc pas pour la psychologie un instrument d'autonomisation et d'unification mais, par la radicalisation qu'elle provoque, elle a conduit à un clivage, selon le modèle biologique auquel on adhère, qui sépare aujourd'hui cognitivisme et behaviorisme pour ne citer que deux des approches les plus représentatives.

Ainsi, si l'on insiste bien souvent sur la rupture qu'aurait opérée le darwinisme dans le champ des sciences de l'homme et en particulier de la psychologie, c'est pour accréditer l'idée que ces disciplines auraient trouvé là l'instrument de leur rupture d'avec la philosophie. Ribot a largement contribué à la creation de ce mythe :

17. Adolf Kussmaul, Untersuchungen über das Seelenleben des Neugeborenen Menschen, Tuibingen, F. Pietzeker, 1859.

18. Wilhelm PreYer, L'Âme de l'enfant, Paris, Félix Alcan, 1887 (1 $1^{\text {re }}$ éd. all., Die Seele des Kindes, Leipzig, L. Fernau, 1881).

19. Emst HAECKEL, Histoire de la création des êtres organisés d'après les lois naturelles, Paris, Reinwald, 1877, p. 359.

20. J. Piaget, Introduction à l'épistémologie génétique. T. III : La Pensée biologique, la pensée psychologique et la pensée sociologique, Paris, P.U.F. 1950.

21. Hans DRIESCH, «Studien über das Regulationsvermögen der Organismen », Wilhelm Roux' Archiv für Entwicklungsmechanik der Organismen. 10, 411, 1900.

22. Paul WEISS, Principles of Development, New York, Holt; 1939. 
" A mesure que s'effaceront des habitudes d'esprit invétérées, on verra de mieux en mieux que la psychologie et la métaphysique, confondues autrefois sous une même dénomination, supposent chacune des aptitudes intellectuelles si opposées qu'elles s'excluent; on comprendra que le talent métaphysique est en raison inverse du talent psychologique; que désormais - à part quelques rares génies qui se rencontreront peut-être - le psychologue doit renoncer à la métaphysique et le métaphysicien à la psychologie $"{ }^{23}$.

E.G. Boring, l'historien officiel de la psychologie expérimentale, a soutenu la même position :

« la psychologie aura tous les avantages à se délivrer complètement de son bagage philosophique, pas seulement dans la pratique mais aussi dans ses présupposés, afin de pouvoir suivre son propre chemin, sans être dérangée par des préoccupations ambiguës $" 24$.

Certes, l'initiative prise par Wundt, en 1879, de fonder à Leipzig un laboratoire de psychologie expérimentale, rapidement imitée en Europe et aux U.S.A., consacre l'institutionnalisation de la psychologie et lui permet d'accéder au rang de discipline universitaire; à ce niveau, mais à ce niveau seulement, il y a donc bien rupture mais les laboratoires qui naissent à l'époque présentent des activités si différentes, animées par des projets si contrastés qu'il est bien difficile de voir dans cette prolifération la naissance d'une science nouvelle et homogène. Analysant l'usage des instruments scientifiques dans les laboratoires de psychologie expérimentale au début du siècle, $F$. Parot $(1988)^{25}$ a montré que des disparités considérables apparaissent entre des pays comme l'Allemagne, la France, les U.S.A. et la Russie tsariste. Ces disparités touchent à la fois les objets étudiés, les méthodes et les conceptions théoriques qui les guident.

Pour nous débarrasser à la fois des mythes de naissance et des mythes de rupture, il nous faudrait interroger l'histoire avec l'ambition de mieux cerner le projet de la psychologie, et son objet. Un regard sur le passé de la psychologie nous montre qu'elle n'a jamais eu d'objet propre : elle a partagé l'âme avec la théologie et la métaphysique, l'entendement

23. Théodule Rıвот, La Psychologie allemande contemporaine, Paris, Félix Alcan, 1879, p. III-IV.

24. E. G. BORING, op. cit. supra n. 1, p. 4.

25. Françoise PAROT, "La psychologie scientifique française et ses instruments au début du $\mathrm{XX}^{\mathrm{c}}$ siècle ", in Études sur l'histoire des instruments scientifiques, Christine BLONDEL et al., eds, Londres, Rogers Turner Books, Ltd, sous presse. 
et la conscience avec la philosophie naturelle et, enfin, le comportement avec la biologie. Toujours placée dans une position subordonnée par rapport à la discipline à laquelle elle faisait son emprunt, elle s'est trouvée également dépendante quant au choix du modèle épistémologique qui sous-tend sa démarche. Quel objet et pour quel projet? Telle devrait être l'interrogation de l'histoire de la psychologie.

Lorsqu'on fait l'histoire d'une discipline, la tentation est grande, et parfois profitable, de chercher à en fixer les commencements. Considérer la psychologie, selon l'étymologie du mot, comme science de l'âme, nous conduit tout droit aux origines du discours philosophique. Mais en réalité ce n'est qu'à la fin du $x^{e}{ }^{e}$ siècle que le mot psychologie apparait sous la plume de quelques théologiens-philosophes allemands de seconde importance. Le mot "psychologie" n'existe pas en grec classique, ni tardif; on ne le trouve pas davantage durant la période byzantine, ni dans la tradition patristique. Le Lexicon de Sophoclès ${ }^{26}$ ne mentionne pas le mot "psuchologia " mais indique plusieurs termes composés à partir du mot "psuchè " qui ne figurent pas tous dans les dictionnaires de grec classique. Ces mots, tels "psuchogonia" (génération de l'âme), "psuchodesmos " (lien), "psuchodiabatos " (passage de l'âme), "psuchodotès" (qui donne l'âme ou la vie) proviennent d'œuvres appartenant, pour la plupart, à la philosophie néoplatonicienne ou gnostique dont on connaît par ailleurs le grand intérêt pour les questions de l'âme, principalement de sa nature et de son devenir. Cet usage, dans la construction des mots, montre bien que l'emploi de la racine "psuchè " est possible et que l'absence de "psuchologia " jusqu'à la fin de la Renaissance indique simplement que l'opposition "anima/animus" était suffisante dans le discours théologique et philosophique.

Si la fin du $\mathrm{xvI}^{e}$ siècle voit l'apparition du mot "psuchologia ", c'est que l'intention est nouvelle. La dérivation d'un terme à partir d'une racine grecque est évidemment banale pour l'époque mais ce qui est plus intéressant, en l'occurrence, c'est la rupture avec une certaine tradition. Qui sont ces théologiens et philosophes allemands qui introduisent cet usage? Rodolphus Goclenius $(1547-1628)^{27}$ est un théologien réformé largement inspiré par Pierre de la Ramée (ou

26. E. A. SophocLes, Greek Lexicon of the Roman and Byzantine Periode 146 BC to $1100 A D$, New York, 1888.

27. Rodolphus GOCLENIUS, PSUCHOLOGI, hoc est hominis perfectione, animo et inprimis ortu hujus, commentationes ac disputationes quorundam Theologorum et Philosophorum nostrae aetatis, quos proxime sequens praefationem pagina ostendit, philosophiae studiosis lectu jucundae et utilitis, Marpurghi, ex officina Egenolphi, 1590. Nous avons consulté la $3^{e}$ édition, de 1597 , seule disponible à la Bibliothèque nationale. 
Ramus) ${ }^{28}$ dont on connaît la totale opposition à Aristote et la contribution au renouveau des études logiques. L'ambition de Goclenius sera de tenter la réconciliation de toutes les philosophies. Son ouvrage Conciliator Philosophicus (1609) ${ }^{29}$ affirme l'unité de la philosophie à travers la multiplicité des écoles. Pour Goclenius, c'est l'identité, chez tous les hommes, du pouvoir de la raison qui constitue la source du commun dénominateur de tous les systèmes philosophiques, au-delà de leurs particularités. C'est donc la tâche de la psychologie que d'étudier cet identique pouvoir de la raison et de l'instituer comme fondement du droit, de la politique et de l'éducation. Otto Cassmann (?-1607), élève de Goclenius, proposera, en 1594, une anthropologie articulée en deux parties : la première "Psychologia " traitera de l'âme, la seconde "Anatomia " décrira le corps ${ }^{30}$. Cette division se perpétuera jusqu'à la fin du XVIIr siècle. Outre cette organisation de la nouvelle "science de l'homme ", Cassmann inscrit son projet dans une perspective sociopolitique qui vise à utiliser la psychologie comme une science de la gestion des individus. Cette science doit reposer sur une connaissance approfondie de la nature humaine, fondement de toute science juridique et sociale ${ }^{31}$.

Une fois que l'on a pris en considération cet acte de naissance lexicale de la psychologie, deux interrogations surgissent. La première questionne les conditions de possibilité de cette science nouvelle : quelle est l'histoire de la rupture de la psychologie avec la tradition scolastique qui comporte, bien entendu, un discours structuré sur l'âme humaine et ses opérations. Cette rupture est encore à décrire. Trois siècles environ séparent la rédaction de la Somme théologique de saint Thomas d'Aquin des écrits de Goclenius et Cassmann, durant lesquels la société, l'économie, la politique et la culture se transforment en profondeur. L'avènement de la psychologie est probablement l'une des résultantes de ces transformations.

La seconde question est relative au destin de la démarche des jusnaturalistes du début du XvII siècle qui ont trouvé une partie de leur inspiration chez Goclenius ou Cassmann. J. Althusius (1557-

28. Pierre DE LA RAMÉ (ou RAMus) dont nous avons consulté Aristotelicae animadversiones, Parisiis, excudebat Jacobus Bogardus, 1543 et la Dialectique, Paris, A. Wechel, 1555.

29. R. Goclenius, Conciliator philosophicus, Cassellis, ex officina Mauritiana, 1609.

30. Otto Cassmann ou Casmanus, Psychologia anthropologica; Sive animae humanae doctrina, methodice informata, capitibus dissecta, singulorumque capitum disquisitionibus, ac controversarum quaestionum ventillationibus illustrata, Hanoviae, impensis P. Fischeri Fr., 1594.

31. O. CASSMANn, Doctrinae et vitae politicae methodicum et breve systema, Francofurti, e collegio musarum Paltheniano, 1603. 
$1638)^{32}$ et $\mathrm{H}$. Grotius (1583-1645) ${ }^{33}$, fondateurs reconnus du droit naturel, ont subi les mêmes influences calvinistes, en théologie, et ramistes, en logique, que nos théologiens-psychologues. Le modèle du droit naturel est rationaliste et laïc (surtout chez Grotius); il pretend fournir une conception générale, non seulement du droit, mais aussi de la politique et de l'éducation. C'est en ce sens qu'il peut être qualifié d'idéologie gestionnaire. Il est certes nécessaire de s'interroger sur les raisons du changement d'objet de la psychologie qui la fera passer de l'étude de la raison ou de l'entendement à celle du comportement mais également sur celles du maintien, dans certains pays, de son projet initial de gestion sociale. Souvenons-nous du "manifeste" behavioriste de J. Watson :

" Si la psychologie suivait le programme suggéré, l'éducateur, le médecin, le juriste et l'homme d'affaires pourrait utiliser nos données de façon pratique pour autant que nous soyons à mème de les obtenir expérimentalement $" 34$.

Bien que l'Allemagne et la France aient été les premières à tenter de fonder le droit sur le savoir psychologique, c'est en Angleterre et aux U.S.A. que la psychologie se développera comme modèle gestionnaire tandis que l'Allemagne et la France s'orienteront, dans un premier temps, vers une psychologie physiologique. Quel est donc le chemin qui conduit du projet des jusnaturalistes à l'impératif behavioriste du contrôle du comportement?

Les interrogations à propos de l'objet de la psychologie, de ses fluctuations historiques sont souvent confrontées à une affirmation qui, elle aussi, fonctionne comme un mythe : l'unité de la psychologie. Quelle que soit la valeur des appels réitérés à cette unité, force est de constater cependant que cette discipline est profondément divisée. Les divisions les plus importantes concernent l'objet, le projet de la psychologie et, enfin, ses modes d'institutionnalisation. La plupart des psychologues s'accordent aujourd'hui à definir le comportement comme objet de la psychologie scientifique. Mais cet objet d'étude n'appartient pas en propre à la psychologie : la sociologie, l'anthropologie, l'ethnologie,

32. Joannes Althusius, Politica methodice digesta, Herbornae Nassoviorum, ex off. C. Corvini, 1603 et Dicaeologicae libri tres, totum et universum jus quo utimur methodice complectantes, cum parallelis hujus et judaici juris, tabulis que insertis, Francofurti, apud

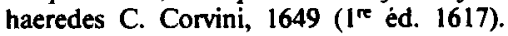

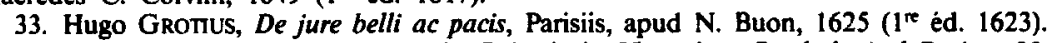

34. John WATSON, "Psychology as the Behaviorist Views it ", Psychological Review, 20, 1913, p. $158-177$ (notre traduction). 
l'économie, etc., étudient elles aussi le comportement des hommes, l'éthologie partage également avec la psychologie l'étude du comportement animal. Ces disciplines sont aujourd'hui largement « décloisonnées ": le psychologue social rencontre le sociologue dans l'étude des comportements collectifs, les méthodes anthropologiques et ethnographiques ne s'appliquent plus seulement aux populations dites « primitives " mais trouvent un large emploi dans les pays les mieux industrialisés. Que la psychologie ne détienne pas le monopole de l'étude du comportement, le fait est incontestable, mais il y a plus. La psychologie est également divisée sur la question du statut méthodologique accordé au comportement. Le comportement est-il l'objet unique et ultime de la recherche ou doit-il, au contraire, être seulement considéré comme la manifestation extérieure, comme le signe indicateur d'une entité, d'un phénomène, d'une structure, d'un processus sous-jacent, inaccessible à l'observation et à l'expérimentation mais qui serait, en tout état de cause, l'objet véritable de la psychologie? Selon la manière de répondre à cette question, on adhère à des conceptions qui s'opposent radicalement sur le plan épistémologique et dont l'histoire elle aussi est à faire. L'opposition du patent et du latent, du manifeste et du caché recouvre partiellement celle de l'externe et de l'interne, celle du sensible et de l'intelligible ou encore celle d'une réalité extérieure conçue comme un donné et de ses multiples représentations. Le positivisme, en proclamant la transparence et l'univocité du monde, nie catégoriquement l'existence d'un niveau latent. Skinner, héritier de cette tradition, formulera cette négation par l'expression: "La peau n'est pas une frontière " ${ }^{35}$; son behaviorisme, conformément à l'impératif nominaliste, dénie toute réalité au monde de la représentation ravalée au rang de simple « flatus vocis ".

Tout au contraire, les partisans de l'autre conception tiennent que toute entreprise de connaissance du monde ou de soi-même comporte au moins deux niveaux de lecture : au premier niveau, on lit des signes apparents dont l'interprétation dévoilera le second niveau. Le comportement n'est alors que le signe ou le symptôme révélateur de l'organisation d'un niveau plus profond, véritable enjeu de l'entreprise de connaissance. Les théories psychologiques qui se réclament de ce second modèle sont nombreuses. La psychologie clinique et la psychopathologie dont la plupart des concepts sont importés de la psychanalyse, la psychologie génétique de J. Piaget, la psycholinguistique chomskyenne et le cognitivisme, pour ne citer que quelques exemples, appartiennent à ce courant qui n'est guère, lui non plus, unitaire. En effet, ces conceptions proposent

35. Burrhus Frederick SkINNER, Science and Human Behavior, New York, Macmillan Comp., 1953, p. 257. 
une organisation en niveaux assez dissemblables; les structures opératoires de Piaget, la structure profonde et la structure de surface du modèle de Chomsky, les schémas computationnels ou les réseaux sémantiques des cognitivistes ne sont guère comparables à premier examen. Cependant toutes font fonctionner des modèles où le comportement, pris comme signe, doit faire l'objet d'une interprétation, qui en dépassant le donné extérieur, construit le contenu et l'organisation du second niveau. C'est de cette herméneutique que doit également rendre compte l'histoire et l'épistémologie de la psychologie. Lorsque la psychologie construit ses objets (âme, idée, raison, entendement, représentation, conscience, inconscient, information, etc.), sa démarche est celle d'une philosophie réaliste; on retrouve donc ici l'opposition du nominalisme au réalisme philosophique. Le lieu du second niveau est habituellement qualifié d'intérieur, de profond, ses opérations se déroulent au-dedans ou en arrière-plan, ses représentations sont sous-jacentes, c'est-à-dire que le second niveau est caractérisé par un jeu de métaphores spatiales se référant toujours et paradoxalement à la «res extensa ». Dire la pensée dans les termes de l'attribut exclusif du corps, voilà chose curieuse pour une discipline qui se réclame, de près ou de loin mais toujours un peu, du cartésianisme.

L'opposition du comportementalisme et du mentalisme a aujourd'hui pour effet de poser certains problèmes de la psychologie d'une façon qui rend les clivages presque inévitables. Dans le domaine de la psychopathologie, la définition de la maladie mentale se trouve bornée par deux questions : "la maladie mentale est-elle mentale?" ${ }^{36}$ et "la maladie mentale est-elle une maladie? $"{ }^{37}$. Dans le domaine de la psychologie de l'enfant, on peut se demander si le monde change autour de l'enfant ou si l'enfant se développe ${ }^{38}$. Dans le domaine du cognitivisme, l'information est-elle un flux d'énergie organisé et porteur de sens qui pénètre dans notre organisme par les différents canaux sensoriels ou est-elle un processus totalement intérieur qui met en forme le monde extérieur ${ }^{39}$ ? Les réponses à ces questions conduisent soit à nier toute intériorité, soit, au contraire, à construire un monde intérieur et à le mettre en communication, au moyen d'un « interface " approprié

36. George WATSON, "Is Mental Illness Mental?", Journal of Psychology, 41, 1956, p. 323-334.

37. Thomas SzaSz, The Myth of Mental Illness, New York, Hoeber Harper, 1961.

38. B. F. SKINNER, Pour une science du comportement : le behaviorisme, Paris/Neuchâtel, Delachaux et Niestlé, 1979, p. 74.

39. Humberto Maturana, Francisco Varela, Autopoiesis and Cognition. The Realization of Living, Dordrecht, D. Reidel Publ. Comp., 1980 ( $1^{\text {re }}$ éd. : De Maquinas y Seres Vivos, Santiago, Editorial Universitaria, 1972), p. XV. 
comme l'indiquent les cognitivistes, avec le monde extérieur. Mais qui communique avec le monde extérieur? De qui fait-on la psychologie? De l'homme, répondra-t-on, puisque la psychologie est science humaine. En fait, la psychologie ne parle jamais de l'homme mais de la personne, de l'individu, du sujet ou encore de l'opérateur. La notion de personne est élaborée par les spirituels du $\mathrm{XII}^{\mathrm{e}}$ siècle à partir de la définition qu'en avait donnée Boèce : «Persona est rationalis naturae individua substantia. " Cette définition sera interprétée à la lumière du dogme trinitaire; la personne possède la mémoire, l'intelligence et la volonté, images respectives du Père, du Fils et de l'Esprit. Cette représentation de la personne, à la fois théologique et psychologique, rejoint les conceptions modernes du courant personnaliste qui n'a pas été sans influence sur la psychologie du $\mathrm{XX}^{\mathrm{e}}$ siècle. A l'opposé, la notion d'individu est intimement associée à la philosophie politique laïque qui se développe à partir du XIVe siècle. Le concept appartient à l'épistémologie de la science moderne dont le paradigme a été défini par Galilée et Newton. L'individu est atome social, élément constitutif de l'organisation sociale dont la psychologie est à construire, afin de mieux le diriger dans le sens de l'intérêt général ou du bien commun. Le $\mathrm{XIX}^{\mathrm{e}}$ siècle verra apparaitre le sujet psychologique, lieu de la conscience avant d'être le support du comportement. Mais le psychologue a oublié que le sujet fut d'abord politique avant d'être logique et grammatical et, enfin, ne plus être que sujet d'expérience. L'expérimentateur le déclarera « sujet percevant ", « sujet pensant ", « sujet agissant », " sujet conscient » et parfois même "sujet humain ", dans les comptes rendus d'expérimentation, pour le distinguer de l'animal avec lequel le darwinisme l'a confondu. Aujourd'hui, l'ergonomie cognitive parle d'opérateur " humain " pour nous distinguer de la machine devenue notre modèle. L'homme entre l'animal et la machine, tel est l'univers dont la psychologie a borné l'étendue.

Sur le plan institutionnel, la psychologie n'est pas plus unitaire. Que de differrences entre son implantation universitaire, son insertion dans les grands organismes de recherche, en particulier au C.N.R.S., et les pratiques sociales des différents métiers de la psychologie. A l'université, la psychologie relève du domaine des sciences humaines, au C.N.R.S., elle appartient au secteur des sciences de la vie. Quant aux " praticiens », ils se répartissent essentiellement entre l'industrie, l'école et l'hôpital. Nous ne savons que peu de chose sur ces modes d'institutionnalisation qui débutent dans la seconde moitié du $\mathrm{XIX}^{\mathrm{e}}$ siècle.

Si l'on s'en tient à la seule histoire de la psychologie française, comment, par exemple, articuler les nombreux sujets de psychologie mis au concours, par l'Académie des sciences morales et politiques, 
à partir de 1860 , sur proposition de Victor Cousin et la creation des premiers laboratoires de psychologie expérimentale dans la dernière décennie du siècle? Le mythe de rupture précédemment évoqué pourrait faire croire qu'il s'agit là de l'œuvre de personnes très différentes : d'un côté, des philosophes rédigent des mémoires sur des thèmes métaphysiques, de l'autre, des expérimentalistes hardis jettent les bases d'un nouveau savoir. Pourtant, A. Binet obtient, en 1887, le prix de l'Académie pour un mémoire, jamais publié, intitulé «La perception extérieure ", sujet mis au concours le 3 juillet $1880^{40}$. Et le même A. Binet intègre, en 1892, le Laboratoire de psychologie physiologique créé par H.E. Beaunis en 1889. Il aura fallu près d'un siècle pour voir déposer aux Archives nationales les documents relatifs à ces établissements et à leurs fondateurs et continuateurs ${ }^{41}$. Le travail des historiens peut commencer.

Il est paradoxal de voir combien les sciences humaines, et en particulier la psychologie, sont aveugles à leur histoire et à leur épistémologie. Il ne s'agit plus, dans une perspective historique et épistémologique, d'interroger l'objet de la psychologie mais bien la psychologie comme objet, c'est-à-dire comme science. Faire ce travail implique tout d'abord de réunir les matériaux de la réflexion : les archives et les textes. Comme nous le déplorions au colloque du C.N.R.S. consacré à l'histoire des sciences de l'homme et de la société, nous ne disposons, aujourd'hui, ni des unes ni des autres. On trouve, ici et là, quelques informations à caractère historique, généralement propos de circonstances prononcés à l'occasion d'un congrès ou d'une commémoration. Ces anecdotes ne peuvent, en aucune façon, tenir lieu de véritable histoire. Nous nous sommes borné à esquisser quelques problématiques, à poser quelques questions qui toutes, ou presque, sont encore sans réponse.

Paul Mengal, Université Paris XII.

\footnotetext{
40. Nous avons découvert mémoire, par hasard, aux Archives de l'Académie des sciences morales et politiques.

41. F. PAROT, "Les archives d'Henri Piéron ", communication à la Journée Archives scientifiques, Centre de recherche en histoire des sciences et des techniques, Cité des sciences et de l'industrie, La Villette, 25 février 1988, à paraitre dans la Gazette des archives. Les archives d'Alfred Binet et d'Henri Wallon sont actuellement en cours de classement.
} 